\title{
Recurrence of ovarian squamous cell carcinoma with MET gene copy number variation: a case report and review of literature
}

\section{Xuhui Dong}

Obstetrics and Gynecology Hospital of Fudan University

\section{Lei Yuan}

Obstetrics and Gynecology Hospital of Fudan University

Liangqing Yao ( $\nabla$ yaoliangqing@163.com )

\section{Case report}

Keywords: ovarian squamous cell carcinoma, optimal cytoreductive surgery, recurrence, MET gene, copy number variation

Posted Date: February 20th, 2020

DOI: https://doi.org/10.21203/rs.2.24118/v1

License: (c) (1) This work is licensed under a Creative Commons Attribution 4.0 International License. Read Full License

Version of Record: A version of this preprint was published at Journal of Ovarian Research on May 31st, 2020. See the published version at https://doi.org/10.1186/s13048-020-00659-y. 


\section{Abstract}

Background: Malignant transformation such as ovarian squamous cell carcinoma (SCC) in ovarian mature cystic teratoma (OMCT) is a rare tumor. The gene mutation of ovarian SCC remains unclear. We herein report a recurrent case of ovarian squamous cell carcinoma with MET gene copy number variation. Case presentation: A 60-year-old woman presented with recurrence of ovarian SCC 8 months after primary surgery. Adhesiolysis, right abdominal wall mass excision, prosthetics, enterectomy, enterostomy and partial cystectomy were performed by laparoscope. Pathologic examination demonstrated metastatic squamous cell carcinoma in ileocecus, rectum and abdominal wall muscle. MET gene copy number was elevated with copy number of six in this case. Postoperatively, the patient was treated with four cycles of combination chemotherapy with docetaxel and carboplatin. The patient was free of disease at 20 months' follow-up. Conclusions: Optimal cytoreductive surgery combined with platinumbased chemotherapy is recommended currently for not only primary tumor but also recurrence. For patients with malignant transformation in OMCT, prompt diagnosis and individualized treatment are crucial for better prognosis. Increased copy number of MET may be correlated with her poor PFS and can be a potential therapeutic target for this case.

\section{Background}

Ovarian mature cystic teratoma (OMCT), which is also called dermoid cyst, is a teratoma of a cystic nature that contains kinds of developmentally mature, solid tissues originating from all three germ-cell layers.[1] The incidence of OMCTs is $1.2-14.2$ cases per 100,000 people per year and $0.14-2 \%$ of them will undergo malignant transformation. More than $80 \%$ of malignant transformations are ovarian squamous cell carcinoma (SCC). [2, 3] Patients with ovarian SCC often had a dismal prognosis and the stage of the disease was an important factor to the prognosis. The 5-year survival rate for all stages was $48.4 \%$, while for adequately staged patients were $75.7,33.8,20.6$ and $0 \%$ respectively. [4] The appropriate treatment for patients with ovarian SCC remains unsolved. We report a case of a woman with recurrence of ovarian SCC in OMCT and review the literature.

\section{Case Presentation}

Medical history

A 60-year-old woman (gravida1, para1) was referred to our hospital because of recurrence of ovarian SCC. She presented with lower abdominal discomfort and transvaginal ultrasonography revealed a $142 * 115 \mathrm{~mm}$ heterogeneous, solid cyst mass in May 2017. Preoperative tumor markers were cancer antigen 125 (CA125): $37 \mathrm{U} / \mathrm{ml}(<35)$ and carcinoembryonic antigen (CEA): $6.18 \mathrm{ng} / \mathrm{ml}(<5)$. Total hysterectomy plus bilateral salpingo-oophorectomy plus omentectomy and iliac lymph node dissection was performed in local hospital due to the malignancy in frozen-section. Pathologic examination indicated right OMCT with malignant transformation into well differentiated SCC; metastases were not 
found in any other excised specimen. The patient was diagnosed as stage IA according to FIGO classification.

Subsequently, 6 cycles of bleomycin, etoposide and cisplatin was provided (etoposide $0.1 \mathrm{~g} \mathrm{~d} 1-5$, cisplatin $40 \mathrm{mg} \mathrm{d} 2-3$, bleomycin 15thousand IU d1-3), and 1 cycle of external beam radiation therapy (EBRT) (DT: $50 \mathrm{~Gy} / 25 \mathrm{~F}$ ) as well. During radiotherapy, a mass with diameter of $2 \mathrm{~cm}$ on right lower abdominal wall was touched. A biopsy specimen showed well differentiated SCC, ovary origin considered. Then she came to our hospital in January 2018.

\section{Auxiliary examination}

Positron emission tomography-computed tomography (PET-CT) showed the elevated uptake of ${ }^{18} \mathrm{~F}-$ Fluorodeoxyglucose (FDG) in right abdominal wall muscle, ileocecus and multiple soft tissue masses around both iliac vessels. (Fig. 1) Elevated tumor marker antigens were human epididymis protein 4 (HE4): $78 \mathrm{pmol} / \mathrm{L}(<74.3)$ and CEA: $6.2 \mathrm{ng} / \mathrm{ml}(<5)$. Other laboratory examinations were within normal values.

Secondary cytoreductive surgery and pathological result

Adhesiolysis, right abdominal wall mass excision, prosthetics, enterectomy, enterostomy and partial cystectomy were performed by laparoscope. 3 tumor masses were detected at the right abdominal wall, ileocecal junction and rectum with a diameter of $3 \mathrm{~cm}, 7 \mathrm{~cm}$ and $4 \mathrm{~cm}$, respectively. Pathologic examination demonstrated metastatic squamous cell carcinoma in ileocecus, rectum and abdominal wall muscle. Immunohistochemically, these cells were diffusely positive for p16, p63, CK-h, Vim and EMA, partly positive for Ki-67 and p53, but negative for CK7.

Whole exome sequencing

The mutation was shown by a circus picture. (Fig. 2) The whole exome sequencing identified 396 somatic mutation events in this case. (Table 1) Two hundred and thirty-nine (60.4\%) were nonsynonymous single nucleotide variation (SNV) and thirty-four were disruptive insertion-deletion events. There were mutations in 7 known driver genes including TP53, CDKN2A, ATR, PTCH1, MCM-7, RAG-1 and SPTAN-1. Mutations in MET, MYC and JAK2 were all synonymous SNV. Exonic mutation in PIK3CA, KMT2A, GNAQ and SMARCA4 were not found in this case. 
Table 1

Classification and number of

mutations in exonic region

\begin{tabular}{|ll|}
\hline Type of mutation & number \\
\hline deletion & 28 \\
\hline insertion & 6 \\
\hline nonsynonymous SNV & 239 \\
\hline stopgain & 12 \\
\hline synonymous SNV & 111 \\
\hline
\end{tabular}

The copy number variation (CNV) was shown in Table 2. Gene amplification in MYC and EGFR which was reported in previous study were also found in this case. Besides, MET gene copy number was elevated with copy number of six which indicated the patient may benefit from c-MET small molecule tyrosine kinase inhibitors such as crizotinib.

Table 2

Number of copy number variation

\begin{tabular}{|lll|}
\hline Copy number & Number & Interval \\
\hline 1 & 1 & 99464 \\
\hline 3 & 316 & 385397789 \\
\hline 4 & 187 & 299076274 \\
\hline 5 & 153 & 388442175 \\
\hline 6 & 139 & 628221903 \\
\hline 7 & 65 & 617883211 \\
\hline 8 & 26 & 379530248 \\
\hline 9 & 5 & 53952072 \\
\hline 11 & 1 & 15567276 \\
\hline 13 & 1 & 100670 \\
\hline
\end{tabular}

Adjuvant treatment and follow up

After the operation, the patient was offered platinum-based chemotherapy for 4 cycles (carboplatin AUC5, docetaxel $75 \mathrm{mg} / \mathrm{m}^{2}$ intravenously). The patient was followed up every 3 months. PET-CT scan in 
July 2019 showed no abnormally elevated uptake of FDG. Up till now, there has been no evidence of tumor recurrence.

\title{
Discussion And Conclusions
}

\author{
Risk factors of malignant transformation in OMCTs
}

Malignant transformation arising from OMCTs is diagnosed with difficulty in the preoperative period. Only $1-2 \%$ of them can be diagnosed preoperatively. We can only depend on some risk factors as follow: i) postmenopausal age; ii) diameter more than $10 \mathrm{~cm}$ or growing rapidly; iii) elevated serum tumor markers especially SCC antigen and CA125; iv) the presence of a solid component that extends transmurally and invades the adjacent structures in magnetic resonance imaging (MRI). [1, 3, 5-7] Cases with these factors usually had a worse prognosis.[4]

Our patient is a postmenopausal woman with a $142 * 115 \mathrm{~mm}$ mass and mildly elevated the level of tumor markers. Before the primary operation, she didn't receive further examination such as CT or MRI. Although the frozen-section found a positive result, more auxiliary examination should be done before the primary surgery.

\section{Treatment of ovarian SCC}

Operation played a pivotal role during both diagnosis and treatment. Patients who received optimal cytoreduction followed with adjuvant chemotherapy seem to have a good outcome with longer survival. $[8,9]$ Optimal cytoreduction can bring a mean survival of 14 months while 7.8 months in suboptimal cases.[10] Kikkawa et al.[6] reported a 79\% 5-year-survival rate for patients without residual disease compared with $10.1 \%$ for those with residual disease. Minimization of tumor burden may improve the effect of adjuvant treatment. Optimal surgical resection can provide a better curative effect in both initial and recurrent cases.

On account of the activity of cisplatin in ovarian cancer and gynaecological squamous-cell carcinomas, chemotherapy with cisplatin and taxane became a common therapy in SCC, and have been extended progression-free interval in most patients.[3, 8, 11, 12] Patients received adjuvant chemotherapy (mostly platinum-based) appeared to survive longer than those who had surgery only. $[4,13]$ Several studies suggested that chemotherapy with BEP can be used in ovarian SCC.[14] Nevertheless our patient had a poor effect to the chemotherapy with BEP, which may lead to the recurrence within a short period of time.

Gene mutation in OMCTs

Several studies showed that malignant transformation of OMCTs may be associated with genetic mutations, high-risk human papillomavirus infection and metaplastic squamous epithelium.[15-19] OMCT-associated SCC had a much higher overall mutational burden and copy number alterations than OMCT. The most frequently altered genes in SCC were TP53 (20/25 cases, 80\%), PIK3CA (13/25 cases, $52 \%)$ and CDKN2A (11/25 cases, 44\%).[20] 
In the study done by Iwasa et al.,[21] overexpression of the p53 protein was observed in $67 \%$ SCC cases (14 in 21 cases), while four of them had point mutations in the p53. In humans, p16 is encoded by the CDKN2A gene. Expression of p16 protein decreased in 86\% patients (18 in 21 cases), and p16 hypermethylation and point mutation was noticed in 6 and 7 respectively. Another study indicated that p53 and p16 expression in SCC were significantly higher than that in the teratomatous skins.[22] P53 and p16-Rb pathways may be related to SCC arising in OMCT. The mutation of TP53 and CDKN2A were also found in our case. Other 5 genes (ATR, PTCH1, MCM-7, RAG-1 and SPTAN-1) were not mentioned in any research about ovarian SCC, although they were demonstrated to be corrected with oncogenesis and chemotherapy resistance in epithelial ovarian cancer or SCC at other sites.

In addition, p16 overexpression are usually caused by the human papillomavirus (HPV). There were reports that HPV infection might be a factor that lead to the malignant transformation. [23] In our case, we also can find diffusely positive for p16 in immunohistochemical staining while her HPV was negative.

Due to low morbidity, it is still not clear to the oncogenesis of SCC in MCT. Because of the tumor heterogeneity, the gene mutation which is the base of targeted therapy in carcinoma with same histological type varies from case to case. Gene sequencing may be a facial step to both diagnosis and treatment. Nowadays personalized cancer medicine becomes more and more important during the treatment in malignancies. Except for treatment mentioned above, targeted therapy is also a way for refractory cases. Increased copy number of MET in our case may be a potential therapeutic target.

MET gene CNV and the targeted therapy

MET gene CNV in ovarian SCC hasn't been reported in previous studies. The MET gene is located on chromosome 7q21-q31 and encodes a receptor tyrosine kinase c-MET for hepatocyte growth factor (HGF).[24][25] When bound by HGF, c-Met is activated by autophosphorylation along with a number of phosphorylated proteins, which in turn phosphorylates and activates several downstream pathways.[24] And then a range of biological effects may present in tumor cells including scattering, branching morphogenesis, cell motility, invasion, migration and eventual metastasis.[26]

Aberrant c-Met activation is associated with cancer progression such as more sites of metastasis and an adverse outcome in many malignant tumers.[27] Epithelial ovarian carcinomas, which mostly consisted in serous cancer, with c-Met expression might be a potential prognostic marker for patients with advanced stage.[28] Patients with high c-Met levels had a shorter median survival compared with those with low c-Met expression (17 months versus 32 months, $P=0.001$ ).[29] To the contrary, another two studies showed high c-Met cases with clear cell carcinoma and mucinous carcinoma had lower stage as well as significantly longer PFS.[30, 31] The prognosis significance of c-MET may be different between type I and type II ovarian cancer.

CNV of MET was a common characteristic in malignant tumor. The high polysomy CNV of the MET gene and was found in $18 \%$ (16/89) clear-cell adenocarcinomas and 1.88\% (3/106) non-clear-cell type ovarian carcinomas.[32] Another study indicated that high polysomy of chromosome 7 was found in $9.5 \%$ 
(10/105) ovarian cancers and most of them were high-grade serous carcinomas.[30] In addition, MET amplification was found in several tumors associated with adverse prognostic factors in carcinoma origin from lung, stomach, gallbladder and ovary. Shorter PFS and OS were observed in cases with increased CNV of MET. Although malignant transformation in OMCTs with an increased CNV of MET had been never reported, her poor PFS met the conclusion mentioned above.

Gene amplification or exon 14 splice site mutations of c-MET in non-small cell lung cancer tumors in which patients may derive benefit from c-MET small molecule tyrosine kinase inhibitors such as crizotinib.[33] Blocking c-Met expression may overcome the resistance of cancer cells to cisplatin.[34] After treatment with crizotinib, cisplatin-induced proliferation inhibition and apoptosis were observed.[35] The long-term efficacy of crizotinib was found in several malignant tumors with MET amplification such as gastric and renal carcinoma.[36, 37] A combination of targeted therapy and chemotherapy may be a trend for tumor treatment.

There have been few reports of targeted therapy to patients with ovarian SCC. Verguts et al.[19] reported a case with positive stain for epidermal growth factor receptor (EGFR), and she was given gefitinib after bone metastasis. Unfortunately, after 4-month-treatment, disease was progressive. Up to now, there were no signs of recurrence in 15-month follow-up. Due to the poor prognosis of patients with ovarian SCC, we hope to take actions to improve her outcome if her disease progressed and didn't reflect to standard treatment. At that time, crizotinib may be an alternative therapy after failed multi-line treatment.

In conclusion, ovarian SCC in OMCT is a rare malignancy. Early detection is crucial to patient survival. Whether a primary or recurrent case, complete surgical resection combined with chemotherapy is one of the most important prognosis factors. Individualized treatment is crucial especially for recurrent cases. After optimal debulking surgery and adjuvant therapy, the patient had a good outcome up to now. Increased CNV of MET can be a potential therapeutic target. Due to the limited data, further analysis is necessary to elucidate the biology and treatment of SCC transformation in OMCT.

\section{Abbreviations}

OMCT: ovarian mature cystic teratoma; SCC: squamous cell carcinoma; CA125: cancer antigen 125; CEA: carcinoembryonic antigen; HE4: human epididymis protein 4 ; EBRT: external beam radiation therapy; PET-CT: positron emission tomography-computed tomography; MRI: magnetic resonance imaging; FDG: Fluorodeoxyglucose; HGF: hepatocyte growth factor; EGFR: epidermal growth factor receptor; CNV: copy number variation

\section{Declarations}

\section{Acknowledgements}

Dr. Hu PC for contributing with patient and imaging selection. 


\section{Funding}

This research did not receive any specific grant.

\section{Availability of data and materials}

The data used or analyzed are all included in this published article.

\section{Authors' contributions}

DXH: data collection, reviewing the literature, drafting and revising the manuscript. YL: case selection and revision of the manuscript. YLQ: case selection and critical review of the manuscript. All authors read and approved the final manuscript.

\section{Ethics approval and consent to participate}

The study received ethics approval from the Commission for Scientific Research in the Obstetrics and Gynecology Hospital of Fudan University.

\section{Consent for publication}

The patient provided written informed consent for the publication of this case report.

\section{Competing interests}

The authors declare that they have no competing interests.

\section{Author details}

${ }^{1}$ Department of Gynecology, Obstetrics and Gynecology Hospital of Fudan University, 128 Shenyang Road, Yangpu District, Shanghai 200090, People's Republic of China. ${ }^{2}$ Department of Gynecology, Obstetrics and Gynecology Hospital of Fudan University, 128 Shenyang Road, Yangpu District, Shanghai 200090, People's Republic of China. ${ }^{3}$ Department of Gynecology, Obstetrics and Gynecology Hospital of Fudan University, 128 Shenyang Road, Yangpu District, Shanghai 200090, People's Republic of China.

\section{References}

1. Goudeli C, Varytimiadi A, Koufopoulos N, Syrios J, Terzakis E: An ovarian mature cystic teratoma evolving in squamous cell carcinoma: A case report and review of the literature. Gynecol Oncol Rep 2017, 19:27-30.

2. Avci S, Selcukbiricik F, Bilici A, Ozkan G, Ozagari AA, Borlu F: Squamous cell carcinoma arising in a mature cystic teratoma. Case Rep Obstet Gynecol 2012, 2012:314535.

3. Hackethal A, Brueggmann D, Bohlmann MK, Franke FE, Tinneberg HR, Munstedt K: Squamous-cell carcinoma in mature cystic teratoma of the ovary: systematic review and analysis of published data. 
Lancet Oncol 2008, 9(12):1173-1180.

4. Chen RJ, Chen KY, Chang TC, Sheu BC, Chow SN, Huang SC: Prognosis and treatment of squamous cell carcinoma from a mature cystic teratoma of the ovary. Journal of the Formosan Medical Association = Taiwan yi zhi 2008, 107(11):857-868.

5. Park SB, Kim JK, Kim KR, Cho KS: Preoperative diagnosis of mature cystic teratoma with malignant transformation: analysis of imaging findings and clinical and laboratory data. Arch Gynecol Obstet 2007, 275(1):25-31.

6. Kikkawa F, Ishikawa H, Tamakoshi K, Nawa A, Suganuma N, Tomoda Y: Squamous cell carcinoma arising from mature cystic teratoma of the ovary: a clinicopathologic analysis. Obstet Gynecol 1997, 89(6):1017-1022.

7. Nanki Y, Chiyoda T, Kataoka F, Nomura H, Nakadaira N, Iwasa N, Hashimoto S, Arima H, Susumu N, Aoki D: Elevated preoperative neutrophil : lymphocyte ratio as a preoperative indicator of mature cystic teratoma with malignant transformation. J Obstet Gynaecol Res 2017, 43(4):744-748.

8. Abhilasha N, Bafna UD, Pallavi VR, Rathod PS, Krishnappa S: A review of squamous cell carcinoma arising in mature cystic teratoma of the ovary. Indian J Cancer 2016, 53(4):612-614.

9. Gadducci A, Giuliani D, Cosio S, Lissoni A, Ferrero AM, Landoni F: Clinical Outcome of Patients With Malignant Tumors Associated With Mature Cystic Teratomas of the Ovary: A Retrospective Multicenter Italian Study. Anticancer research 2019, 39(5):2513-2517.

10. Sakuma M, Otsuki T, Yoshinaga K, Utsunomiya H, Nagase S, Takano T, Niikura H, Ito K, Otomo K, Tase $T$ et al: Malignant Transformation Arising From Mature Cystic Teratoma of the Ovary. International Journal of Gynecological Cancer 2010, 20(5):766-771.

11. Rim SY, Kim SM, Choi HS: Malignant transformation of ovarian mature cystic teratoma. International journal of gynecological cancer : official journal of the International Gynecological Cancer Society 2006, 16(1):140-144.

12. Chiang AJ, Chen MY, Weng CS, Lin H, Lu CH, Wang PH, Huang YF, Chiang YC, Yu MH, Chang CL: Malignant transformation of ovarian mature cystic teratoma into squamous cell carcinoma: a Taiwanese Gynecologic Oncology Group (TGOG) study. Journal of gynecologic oncology 2017, 28(5):e69.

13. Roxburgh P, Glasspool R: Squamous carcinoma of the ovary. Current oncology reports 2014, 16(12):413.

14. Li C, Zhang Q, Zhang S, Dong R, Sun C, Qiu C, Zhang Z, Yang X, Kong B: Squamous cell carcinoma transformation in mature cystic teratoma of the ovary: a systematic review. BMC cancer 2019, 19(1):217.

15. Iwasa A, Oda Y, Kaneki E, Ohishi Y, Kurihara S, Yamada T, Hirakawa T, Wake N, Tsuneyoshi M: Squamous cell carcinoma arising in mature cystic teratoma of the ovary: an immunohistochemical analysis of its tumorigenesis. Histopathology 2007, 51(1):98-104.

16. Mai KT, Yazdi HM, Bertrand MA, LeSaux N, Cathcart LL: Bilateral primary ovarian squamous cell carcinoma associated with human papilloma virus infection and vulvar and cervical intraepithelial 
neoplasia. A case report with review of the literature. Am J Surg Pathol 1996, 20(6):767-772.

17. Manolitsas TP, Lanham SA, Hitchcock A, Watson RH: Synchronous ovarian and cervical squamous intraepithelial neoplasia: an analysis of HPV status. Gynecol Onco/ 1998, 70(3):428-431.

18. Tate G, Tajiri T, Suzuki T, Mitsuya T: Mutations of the KIT gene and loss of heterozygosity of the PTEN region in a primary malignant melanoma arising from a mature cystic teratoma of the ovary. Cancer Genet Cytogenet 2009, 190(1):15-20.

19. Verguts J, Amant F, Moerman P, Vergote I: HPV induced ovarian squamous cell carcinoma: case report and review of the literature. Arch Gynecol Obstet 2007, 276(3):285-289.

20. Cooke SL, Ennis D, Evers L, Dowson S, Chan MY, Paul J, Hirschowitz L, Glasspool RM, Singh N, Bell S et al: The Driver Mutational Landscape of Ovarian Squamous Cell Carcinomas Arising in Mature Cystic Teratoma. Clin Cancer Res 2017, 23(24):7633-7640.

21. Iwasa A, Oda Y, Kurihara S, Ohishi Y, Yasunaga M, Nishimura I, Takagi E, Kobayashi H, Wake N, Tsuneyoshi M: Malignant transformation of mature cystic teratoma to squamous cell carcinoma involves altered expression of p53- and p16/Rb-dependent cell cycle regulator proteins. Pathol Int 2008, 58(12):757-764.

22. Zhu HL, Zou ZN, Lin PX, Li WX, Huang YE, Shi XX, Shen H: Malignant transformation rate and p53, and p16 expression in teratomatous skin of ovarian mature cystic teratoma. Asian Pacific journal of cancer prevention : APJCP 2015, 16(3):1165-1168.

23. Chiang AJ, Chen DR, Cheng JT, Chang TH: Detection of human papillomavirus in squamous cell carcinoma arising from dermoid cysts. Taiwanese journal of obstetrics \& gynecology 2015, 54(5):559-566.

24. Mo HN, Liu P: Targeting in cancer therapy. Chronic diseases and translational medicine 2017, 3(3):148-153.

25. Jung KH, Park BH, Hong SS: Progress in cancer therapy targeting c-Met signaling pathway. Archives of pharmacal research 2012, 35(4):595-604.

26. Maulik G, Shrikhande A, Kijima T, Ma PC, Morrison PT, Salgia R: Role of the hepatocyte growth factor receptor, c-Met, in oncogenesis and potential for therapeutic inhibition. Cytokine \& growth factor reviews 2002, 13(1):41-59.

27. Jardim DL, Tang C, Gagliato Dde M, Falchook GS, Hess K, Janku F, Fu S, Wheler JJ, Zinner RG, Naing A et al: Analysis of 1,115 patients tested for MET amplification and therapy response in the MD Anderson Phase I Clinic. Clinical cancer research : an official journal of the American Association for Cancer Research 2014, 20(24):6336-6345.

28. Ayhan A, Ertunc D, Tok EC, Ayhan A: Expression of the c-Met in advanced epithelial ovarian cancer and its prognostic significance. International journal of gynecological cancer: official journal of the International Gynecological Cancer Society 2005, 15(4):618-623.

29. Sawada K, Radjabi AR, Shinomiya N, Kistner E, Kenny H, Becker AR, Turkyilmaz MA, Salgia R, Yamada SD, Vande Woude GF et al: c-Met overexpression is a prognostic factor in ovarian cancer 
and an effective target for inhibition of peritoneal dissemination and invasion. Cancer research 2007, 67(4):1670-1679.

30. Kim WY, Shim SH, Jung HY, Dong M, Kim SN, Lee SJ: The gene copy number of c-MET has a significant impact on progression-free survival in Korean patients with ovarian carcinoma. Human pathology 2017, 64:98-105.

31. Lim L, Wu CC, Hsu YT, Sun FJ, Chang CL: Clinical significance of c-Met and phospho-c-Met (Tyr1234/1235) in ovarian cancer. Taiwanese journal of obstetrics \& gynecology 2019, 58(1):105110.

32. Yamamoto S, Tsuda H, Miyai K, Takano M, Tamai S, Matsubara O: Gene amplification and protein overexpression of MET are common events in ovarian clear-cell adenocarcinoma: their roles in tumor progression and prognostication of the patient. Modern pathology : an official journal of the United States and Canadian Academy of Pathology, Inc 2011, 24(8):1146-1155.

33. Paik PK, Drilon A, Fan PD, Yu H, Rekhtman N, Ginsberg MS, Borsu L, Schultz N, Berger MF, Rudin CM et al: Response to MET inhibitors in patients with stage IV lung adenocarcinomas harboring MET mutations causing exon 14 skipping. Cancer discovery 2015, 5(8):842-849.

34. Li E, Hu Z, Sun Y, Zhou Q, Yang B, Zhang Z, Cao W: Small molecule inhibitor of c-Met (PHA665752) suppresses the growth of ovarian cancer cells and reverses cisplatin resistance. Tumour biology : the journal of the International Society for Oncodevelopmental Biology and Medicine 2016, 37(6):78437852.

35. Wang J, Cheng JX: c-Met inhibition enhances chemosensitivity of human ovarian cancer cells. Clinical and experimental pharmacology \& physiology 2017, 44(1):79-87.

36. Hou GX, Song BB: Gastric cancer patient with c-MET amplification treated with crizotinib after failed multi-line treatment: A case report and literature review. Math Biosci Eng 2019, 16(5):5923-5930.

37. Rochigneux P, Thomassin-Piana J, Laibe S, Brunelle S, Salem N, Escudier B, Vassal G, Gravis G: Longterm efficacy of crizotinib in a metastatic papillary renal carcinoma with MET amplification: a case report and literature review. BMC Cancer 2018, 18(1):1159.

\section{Figures}




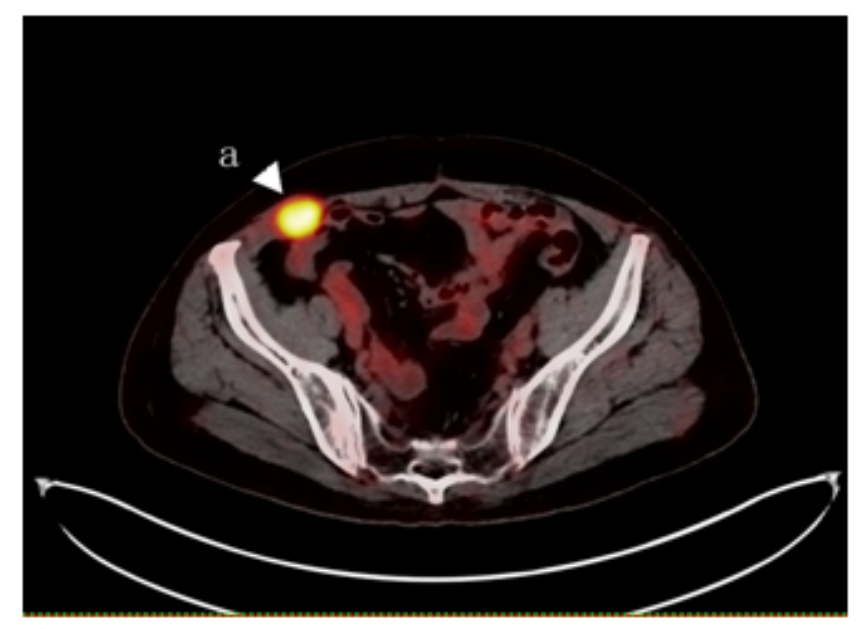

Fig. 1a

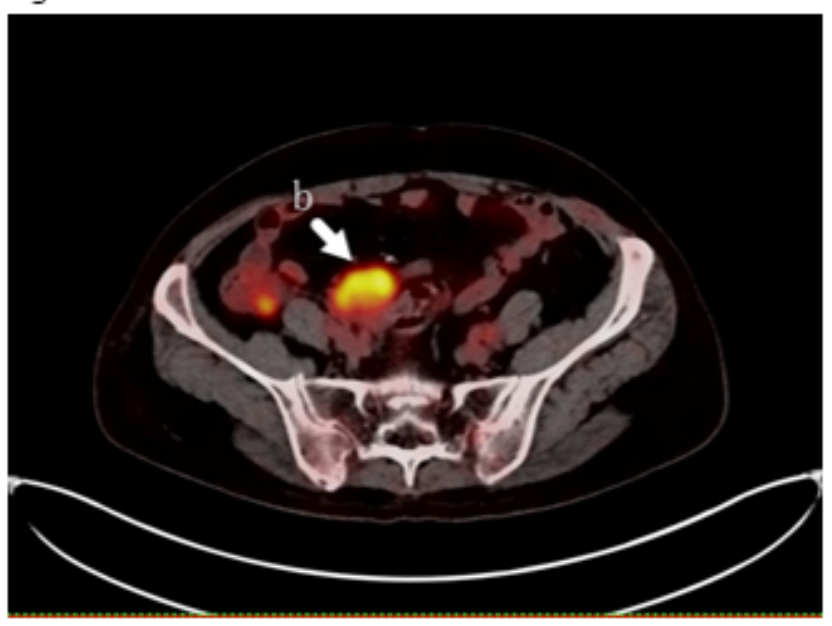

Fig. 16

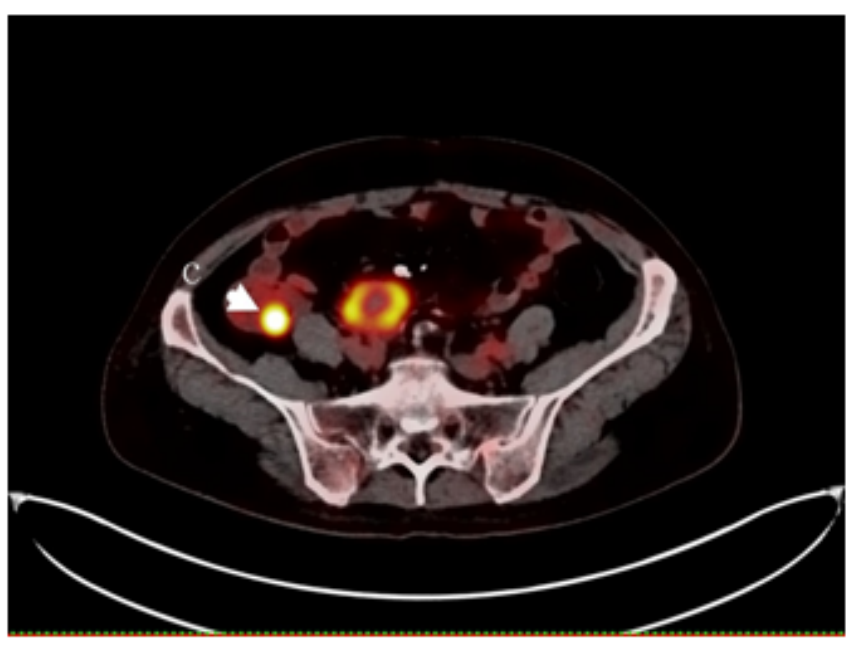

Fiq. 1c

Figure 1

PET-CT fingdings. The elevated uptake of FDG in the right abdominal wall (a), multiple soft tissue masses around both iliac vessels (b) and ileocecus (c). 


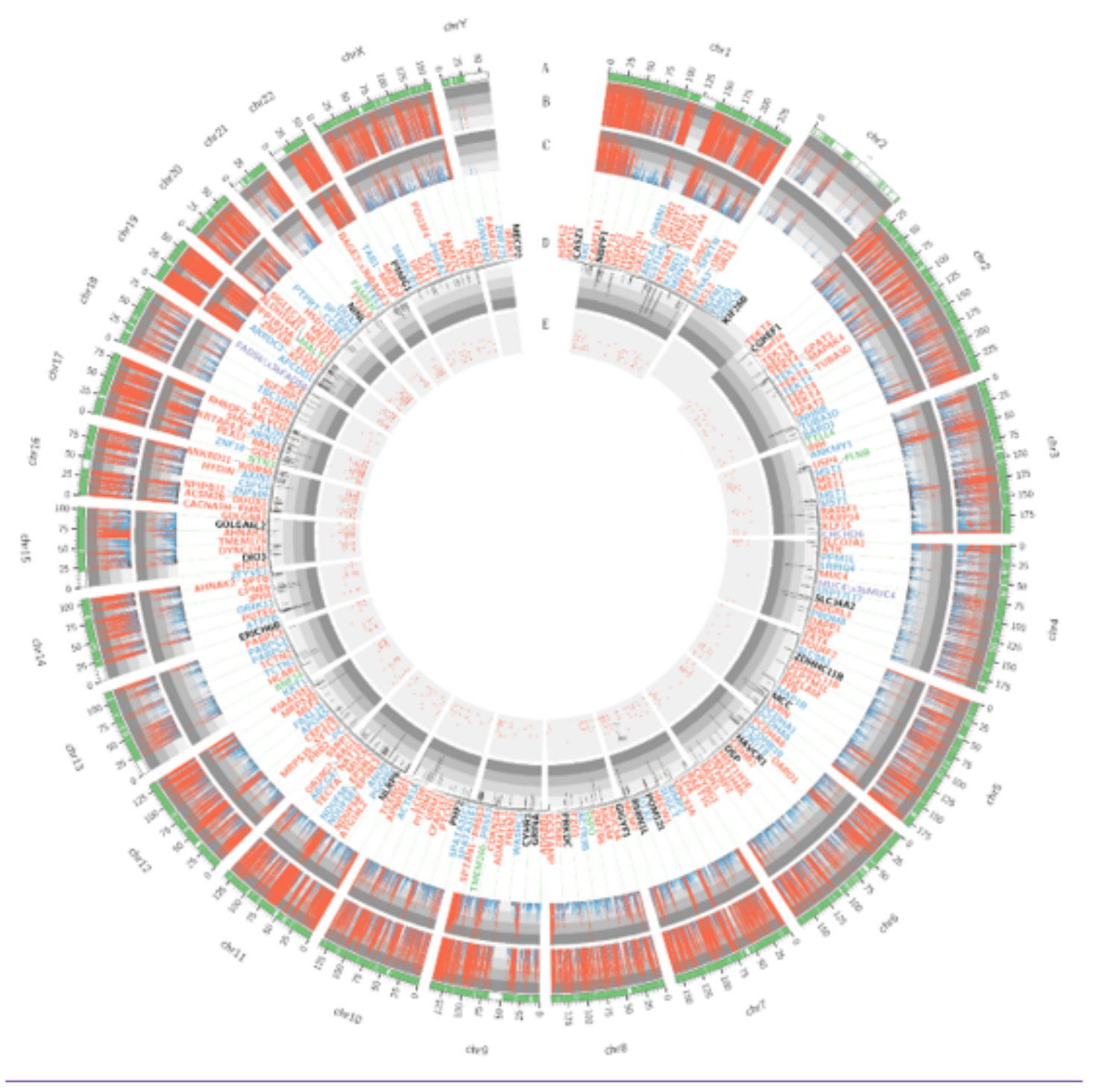

Figure 2

Summary of exome sequencing results displayed as a Circos diagram. Each circle from the outer to inner is: A) trapping area; B) sequencing depth of tumor samples: different color represented various depth: red one $>=500$, blue one $>=100$, others are black; C) sequencing depth of control group samples; D) frequency of somatic mutation and gene related to exonic and splicing mutation: splice site mutation, synonymous mutation, nonsynonymous mutation and terminator codon mutation are purple, blue, red and green respectively. E) somatic cell CNV: various color represents different copy number: red one $>2$, black one $=2$ and blue $=1$. 